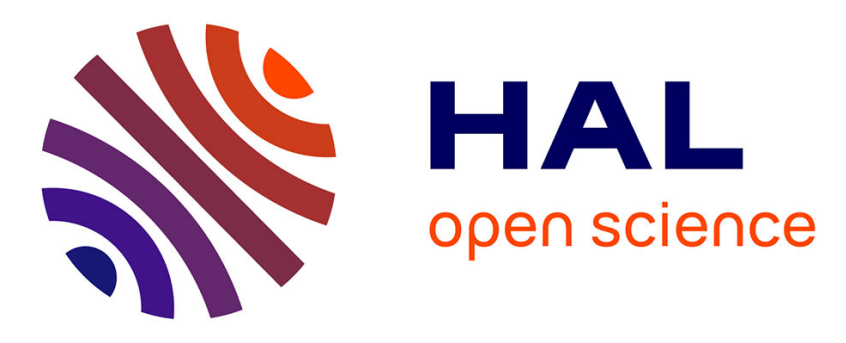

\title{
Optimizing charging station locations for urban taxi providers
}

Johannes Asamer, Martin Reinthaler, Mario Ruthmair, Markus Straub, Jakob Puchinger

\section{- To cite this version:}

Johannes Asamer, Martin Reinthaler, Mario Ruthmair, Markus Straub, Jakob Puchinger. Optimizing charging station locations for urban taxi providers. Transportation Research. Part A, General, 2016, 85, pp.Pages 233-246. 10.1016/j.tra.2016.01.014 . hal-01222597v2

\section{HAL Id: hal-01222597 \\ https://hal.science/hal-01222597v2}

Submitted on 19 Jan 2016

HAL is a multi-disciplinary open access archive for the deposit and dissemination of scientific research documents, whether they are published or not. The documents may come from teaching and research institutions in France or abroad, or from public or private research centers.
L'archive ouverte pluridisciplinaire $\mathbf{H A L}$, est destinée au dépôt et à la diffusion de documents scientifiques de niveau recherche, publiés ou non, émanant des établissements d'enseignement et de recherche français ou étrangers, des laboratoires publics ou privés. 


\title{
Optimizing Charging Station Locations for Urban Taxi Providers
}

\author{
Johannes Asamer ${ }^{\mathrm{a}, *}$, Martin Reinthaler ${ }^{\mathrm{a}}$, Mario Ruthmair ${ }^{\mathrm{a}, \mathrm{b}}$, Markus Straub $^{\mathrm{a}}$, \\ Jakob Puchinger ${ }^{\mathrm{a}, \mathrm{c}, \mathrm{d}}$ \\ ${ }^{a}$ Mobility Department, Austrian Institute of Technology, Vienna, Austria \\ ${ }^{b}$ Department of Statistics and Operations Research, University of Vienna, Austria \\ ${ }^{c}$ Laboratoire Genie Industriel, CentraleSupélec, Université Paris-Saclay, France \\ ${ }^{d}$ Institut de Recherche Technologique SystemX, Palaiseau, France
}

\begin{abstract}
Electric vehicles are gaining importance and help to reduce dependency on oil, increase energy efficiency of transportation, reduce carbon emissions and noise, and avoid tail pipe emissions. Because of short driving distances, high mileages, and intermediate waiting times, fossil-fuelled taxi vehicles are ideal candidates for being replaced by battery electric vehicles (BEVs). Moreover, taxis as BEVs would increase visibility of electric mobility and therefore encourage others to purchase an electric vehicle. Prior to replacing conventional taxis with BEVs, a suitable charging infrastructure has to be established. This infrastructure, which is a prerequisite for the use of BEVs in practice, consists of a sufficiently dense network of charging stations taking into account the lower driving ranges of BEVs.
\end{abstract}

In this case study we propose a decision support system for placing charging stations to satisfy the charging demand of electric taxi vehicles. Operational taxi data from about 800 vehicles is used to identify and estimate the charging demand for electric taxis based on frequent origins and destinations of trips. Next, a variant of the maximal covering location problem is formulated and solved, aiming at satisfying as much charging demand as possible with a limited number of charging stations. Already existing fast charging locations are considered in the optimization problem. In this work, we focus on finding regions in which charging stations should be placed, rather than exact locations. The exact location within an area is identified in a post-optimization phase (e.g., by authorities), where environmental conditions are considered, e.g., the capacity of the power network, availability of space, and legal issues.

Our approach is implemented in the city of Vienna, Austria, in the course of an applied research project conducted in 2014. Local authorities, power network operators, representatives of taxi driver guilds as well as a radio taxi provider participated in the project and identified exact locations for charging stations based on our decision support system.

\footnotetext{
* Corresponding author

Email addresses: johannes.asamer@ait.ac.at (Johannes Asamer), martin.reinthaler@ait.ac.at (Martin Reinthaler), mario.ruthmair@univie.ac.at (Mario Ruthmair), markus.straub@ait.ac.at (Markus Straub), jakob.puchinger@centralesupelec.fr (Jakob Puchinger)
} 
Keywords: taxi, electric vehicles, charging infrastructure, optimization, maximal covering location problem

\section{Introduction}

Electrification of vehicles is a viable way to achieve clean and efficient transportation. It helps to reduce dependency on oil, avoid tail pipe emissions, reduce carbon emissions and noise, and increase energy efficiency of transportation. Different types of (hybrid) electric vehicles are already available on the market and charging infrastructure is going to be established or extended. Among electric vehicles, battery electric vehicles (BEVs) have the highest potential for achieving clean transportation, since there are no tailpipe emissions or additional weight due to an auxiliary combustion engine. Major drawbacks of pure electric vehicles are higher acquisition costs and limited driving ranges due to limited energy storage capabilities.

Although BEVs have much lower driving ranges compared to ICEVs, this is not a problem when trips are below the maximum driving range. Therefore, taxi vehicles are considered as primary candidates for being replaced by BEVs: A high annual mileage accelerates the amortization of the BEV and almost all trips are far below the maximum driving range. Apart from economic and ecological advantages, BEVs as taxis will increase visibility of electric mobility and thus encourage people in utilizing BEVs instead of ICEVs.

In order to emphasize the suitability of BEVs as taxis, we analysed operational data obtained from a taxi dispatch center. Positioning data of approximately 800 taxi vehicles (currently ICEVs) from one radio taxi provider are used for analysing driving patterns and determining a virtual charging demand. According to Egbue and Long (2012) a proper charging infrastructure is critical for the development of an electric vehicle system. In this study we present a method for placing a predefined limited number of charging stations (CSs) while maximizing the coverage of the estimated charging demand of taxi BEVs.

To convince drivers and taxi enterprises to employ BEVs as taxis any negative impact on daily taxi business has to be avoided. This might occur when the vehicle has to be charged and meanwhile is not able to accept a customer trip. Therefore, waiting times between consecutive customer trips should be used for charging. As in other cities, taxis in Vienna are allowed to use taxi stands when waiting for a customer. In this study we define the time difference between arriving and leaving the taxi stand as the time waiting for a customer. The number of charging operations should be kept as low as possible, because each time the driver has to search for a CS and frequently plugging and unplugging to a CS is inconvenient. Moreover, to minimize downtimes of taxi BEVs the usage of fast charging stations is favoured.

We aim to find locations for new fast CSs while also considering already existing fast CSs. Charging demand is determined based on a large amount of operational data and intermediate waiting times at taxi stands are used for charging. When searching for optimal locations, we select regions instead of certain taxi stands. For a region, it is up to an expert (or authority) to decide where to exactly place the CS inside the region. Therefore, the result of the optimization process is rather a support for human decision makers instead of making an obligatory choice. Based on the recommended region an expert can 
choose specific locations for CSs based on different criteria (power supply, open space, etc.).

The key contribution of this study is the description of a decision support system for placing fast CSs. The system is designed to meet requirements of taxi vehicles and was implemented on the basis of real operational data. Existing fast CSs are considered in the algorithm which allows a step-by-step improvement of the charging infrastructure. This study is intended to support authorities to enable a taxi service with BEVs and promote an environmental friendly transportation mode. In cooperation with an electricity provider, a predefined number of CS is installed and optimal locations are found by the method developed in this study. The number of CS is a trade-off between budget constraints on the one hand and coverage of charging demand and road network on the other hand. In this sense, the study at hand provides a method for finding this trade-off based on requirements of authorities (promote electric mobility), electricity provider (cover charging demand) and taxi drivers (reliable and seamless charging infrastructure).

All efforts described here were implemented during a cooperative research project: Project W-ETaxi aims at introducing taxi electric vehicles in Vienna, Austria, involving different stakeholders in order to achieve an optimal and widely accepted result. In a possible follow up of this project, several CSs will be installed and BEVs purchased. Investigations are applied for the Nissan eNV200 electric vehicle. This BEV has a maximum driving range of $170 \mathrm{~km}$, is used in other cities too (e.g., London, New York City) and is therefore a promising candidate for acting as a taxi.

The paper is organized as follows: We discuss the state of the art regarding siting and sizing CSs in Section 2. In Section 3 first we give a description of the data used in this study and how charging demand is derived. Second, the method for finding locations for CSs embedded in a decision support system is described. In Section 4 we present corresponding experimental results and summarize all findings and give recommendations for future research activities in Section 5.

\section{Literature review}

In order to enable a seamless operation of electric vehicles, a charging infrastructure has to be established. In this context research activities regarding the optimal implementation of charging infrastructure gain in importance.

A general overview on related facility location problems and the corresponding state-of-the-art solution approaches can be found in Owen and Daskin (1998) and Daskin (2011). The location problem considered in this article is based on the so-called maximal covering location problem (Church and ReVelle, 1974) maximizing the covered demand subject to a limited number of facilities.

Wang et al. (2013) formulates the problem of optimally planning CSs as a classical facility location and sizing problem. This involves the location of CSs as well as the number of charging points per CS to meet the charging demand of existing and future electric vehicles. The objective is to ensure a seamless supply for BEVs, realized by minimizing distances to CSs and avoiding low waiting times at the CSs. For some applications also the investment and maintenance cost of CSs as well as energy costs are considered in the optimization problem. 
For building a charging infrastructure, an important step is to identify the demand for charging BEVs. Ge et al. (2011) consider traffic flows on road links and a certain percentage of BEVs in relation to the total amount of vehicles. The objective is to minimize energy and travel time losses of BEVs on their way to the next CS. The corresponding optimization problem is solved heuristically by applying a genetic algorithm.

Traffic flows of BEV on intersections and a certain energy consumption rate are used as an indicator for BEV charging demand by Wang et al. (2013); Gao and Guo (2013). Data from household travel surveys is used by Chen et al. (2013) for estimating a parking demand. Locations with a high parking demand are considered as potential locations for CSs. Lingfeng et al. (2010) simply consider the number of residents to estimate the charging demand. In all of these studies the demand is related to intersections which is satisfied if a CS is located at the specific location.

ElBanhawy et al. (2014) present a multi-agent traffic simulation in which each agent moves in the network between a predefined origin-destination (OD) pair and locations of CSs are assumed. The energy consumption per distance unit and a lower threshold on the state of charge (SOC) determine when an agent is in need of being charged. In this case the shortest path starting from the current position with an intermediate stop at the nearest CS and ending at the destination is computed. The authors recommend this approach for assisting in finding locations for CSs, i.e., to assess the impact of a given charging infrastructure.

Similar to this, Hess et al. (2012) estimate the charging demand for virtual trips between randomly selected OD pairs in the first district of Vienna, Austria. Trips are generated by a microscopic traffic simulation (called SUMO) and the decline of the SOC of virtual vehicles is constantly monitored. If the SOC falls below a threshold the BEV is heading for the closest CS, causing additional travel time. The objective is to minimize the additional travel times by optimally selecting locations for a predefined number of CSs. Since the trip data is randomly generated the practical applicability of this approach cannot be guaranteed.

Andrews et al. (2013) use the data from household surveys and estimate whether or not a series of trips can be accomplished by BEVs. For all vehicles which are not able to complete their trips and therefore need additional charge, the distances to the nearest CSs are minimized. This is done by optimally selecting a predefined number of CSs out of a pool of candidate locations. However, this approach assumes that a home-charging possibility for all BEVs is available. In Vienna a considerable amount of inhabitants has no access to home charging due to limited space. Home CSs for private purposes are normally level I, see Table 1, causing long charging times $(>10 \mathrm{~h}$ ). As many taxis are operated 24 hours per day by different drivers, level I charges are not suitable here.

A quite detailed model for optimizing the locations of CSs is presented by He et al. (2013). They modelled the interactions between the power network, the availability of CSs, destination choices, and prices for electricity. One of their assumption is that the driver's decision for choosing a CS depends on the price of electricity. In Vienna the price for electricity does not vary between different locations but depends on the energy provider. Additionally, because of the high complexity of the model, the calibration is expected to be complex and training data difficult to obtain. 
Nie and Ghamami (2013) minimize the sum of battery costs and CS construction costs in order to meet a given level of service. Moreover, the authors focus on long distance trips along a corridor and therefore this study is not comparable to the introduction of BEVs in urban areas.

In contrast to previous studies, we study the optimization of CSs for taxi BEVs and the charging demand is obtained from operational taxi data.

Previous studies (cf. Reinthaler et al., 2014; Sellmair and Hamacher, 2014; Jung et al., 2012) show that BEVs are well qualified as taxis, especially in urban areas. A high mileage together with lower operational costs compensates higher investment costs. Trips can be accomplished without running out of electric energy, and intermediate waiting times can be used for charging.

Neither traffic flows nor the number of residents provide information whether the driver of a BEV is currently busy or has time to charge. From operational taxi data this information can be estimated and used for the placement of CSs. In Cai et al. (2014) mobility data of 11,000 taxis in Beijing, China, is used for estimating a public charging demand. Three indicators (number of vehicles, average waiting time, sum of waiting times) are estimated based on taxi data within the service range $(1.6 \mathrm{~km})$ of gas stations. For each indicator a predefined number of gas stations for mounting a CS are selected based on the ranked indicator score. Since the service range of gas stations may overlap a redundant coverage of charging demand may be possible. In our approach we assign charging demand to disjunct areas and multiple coverages of charging demand can be controlled in the optimization method. Furthermore, Cai et al. (2014) assume hybrid electric vehicles (HEVs) as taxis. For all three indicators a percentage of "electrified miles" and resulting emission reductions are estimated and compared. Thus, the authors focus on investigating the impact of HEVs (with driving ranges comparable to $\mathrm{ICEVs}$ ), where our objective is to enable a taxi service with BEVs only.

Using taxi BEVs for advance reservations in Singapore was investigated by Wang and Cheu (2013). The goal is to combine multiple trips in order to be accomplished by a BEV while minimizing the number of BEVs in the system. Placing CSs is not subject of their work, so CS locations are defined a priori.

To the best of our knowledge, only one study discusses the placement of CSs for taxi BEVs: In Sellmair and Hamacher (2014) only taxi stands are considered as possible locations for CSs. By using simulation techniques, trips between two taxis stands (OD pair) containing a customer trip are generated. Probabilities for assigning an OD pair to a vehicle is determined on the basis of GPS records from five conventional taxis in Munich, Germany. For all taxi BEVs in the simulation, the remaining SOC is used for deciding whether an assigned trip can be accomplished or not. If the SOC is insufficient, the vehicle has to remain at the taxi stand for charging. In case there is no CS at the taxi stand, proposed trips have to be refused three times before moving to another taxi stand containing a CS. On the one hand, increasing the number of CSs will increase the mileage of taxi BEVs (and therefore the earnings), because less trips have to be refused. On the other hand, each additional CS increases fixed and variable costs of the charging infrastructure. The goal is to find an optimal number of CSs for each taxi stand in order to maximize the economical benefit. The simulation neglects that taxi BEVs with a low SOC would immediately head for a taxi stand with a CS. Also, it is unlikely that taxis are refusing three times a customer trip before changing to a taxi stand with CS. In our 


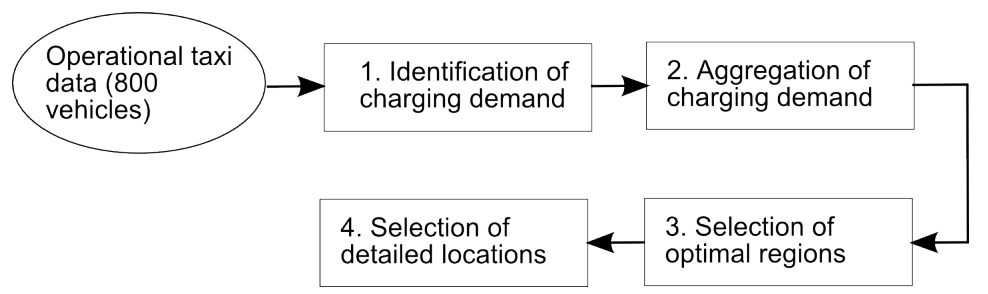

Figure 1: Work flow of decision support system.

approach we assume that the driver is heading for a CS in case of a low SOC. Therefore, CSs have to be placed in areas where a subsequent customer trip can be expected. Sellmair and Hamacher (2014) argue that the optimization of all 215 taxi stands at once is computationally too intensive. Thus, they start with only ten taxi stands and iteratively add further ten taxi stands where five of them are those with the highest economic benefit in the previous iteration and the rest is randomly selected.

In our study we derive the charging demand directly from operational data of a large taxi fleet. Therefore, the distribution of origins and destinations of trips can be deduced directly from the data, without applying a (traffic) simulation. Moreover, we consider the whole area covered by Viennese taxis and a preselection of locations is not necessary. As discussed above, previous approaches recommend a specific location for placing a CS. However, detailed information about environmental conditions at specific locations (e.g., capacity of the power network, legal issues, etc.) might not be available in the planning phase. Moreover, obtaining these details for all considered locations might not be possible in a reasonable amount of time. Therefore, we established an approach more related to practice by recommending a region for placing a CS. The effort of selecting an exact location is therefore limited to a few areas. Furthermore, we aim to find locations for a limited number of CSs solely dedicated to taxis. Cost-efficiency for operating the CSs is not an objective here.

\section{Methodology}

In this section we describe our approach for finding suitable locations for CSs. We start with the description of movement data from about 800 taxi vehicles in Vienna, Austria, and how the charging demand is derived. Demand is afterwards aggregated for predefined cells and handed over to the optimization method. The optimization result is a list of cells suitable for placing a CS. The corresponding work flow is visualized in Fig. 1.

\subsection{Operational taxi data}

In this study charging infrastructure is optimized for taxi BEVs, and therefore we use operational data from a taxi dispatch center to estimate charging demands and possible charging locations. All vehicles are dispatched by a radio taxi provider in Vienna, Austria. A request for a taxi trip placed by a customer over telephone or internet is forwarded to taxi at the three nearest taxi stands. The order is commissioned to the driver first confirming the order. In this sense it is advantageous for a driver to wait in areas with many starting locations of 
customer trips. For dispatching, the location of all taxis registered to the taxi dispatch center (approximately 800) is polled at an interval in the range from 30 to 45 seconds. Additionally, a vehicle status is reported, describing whether the taxi is occupied, available or on the way to a customer. In case a driver finishes a shift, he/she unsubscribes from the system and the new driver registers himself/herself, which means the start and end of a taxi shift can be determined by the radio taxi provider. Position and status of taxis is collected and used primarily for estimating link travel times and detecting traffic incidents. A detailed description of the system can be found in Toplak et al. (2010). For the purpose of link travel time estimation the duration of shifts is not relevant and therefore not provided by the radio taxi provider. However, in an earlier research project with a focus on the impact of a fully electrified taxi fleet on the energy grid (Schuster et al., 2012) all operational taxi data from 2011 were enriched with shift durations and waiting times at taxi stands. This dataset from 2011 is used to demonstrate the suitability of taxi BEVs and to decide which CS terminal type to be used. On the other hand, the charging demand considered here is derived only from vehicle positions and states and therefore a more recent dataset from 2014 could be used.

Although all used taxi data come from the same radio taxi provider, their spatial and temporal distribution is representative for the entire taxi fleet in Vienna. First, 800 taxis are approximately $25 \%$ of all taxis and therefore the sample size is sufficiently large. Second, all Viennese taxis have a licence for the whole city and are using the same taxi stands and other facilities. There is no intended subdivision of the business area by the different radio taxi providers or drivers. Third, the radio taxi provider has no influence on the distribution of the taxis. Orders are forwarded to all drivers waiting at the three taxis stands nearest to the location of the request, but nobody is forced to accept a specific order.

\subsection{Identification of charging demand}

Our estimation of charging demands is based on two aspects: i) When would be a suitable time for charging, and ii) where are associated locations most frequently? Charging during a customer trip has to be avoided, so we have to consider the times and locations between deboarding and boarding. After dropping off the customer the decision which taxi stand is headed for is made solely by the driver based on experience, distance, and utilization of the taxi stand. Therefore, it would be convenient for the driver to have a CS in the vicinity of the taxi stand he/she chooses. On the other hand it would be preferable for the driver to be near to the next customer after the vehicle has been charged. In this context we consider start and end locations of trips as suitable locations for charging from the perspective of an individual driver. By this consideration, travel times between the location where a BEV is in need of charging and the CS will be minimized. In our case we have no information about the energy consumption or SOC along a trip. We assume the chance for a BEV to be in need of charging to be equal for each start/end location. Therefore in regions with many start/end locations more BEVs will search for a CS, i.e., a higher charging demand is expected.

Since charging demand is estimated on the basis of ICEV taxis, the question arises, whether the driving behaviour is comparable to BEVs and what the implications on charging demand are. Origins and destinations of trips are 


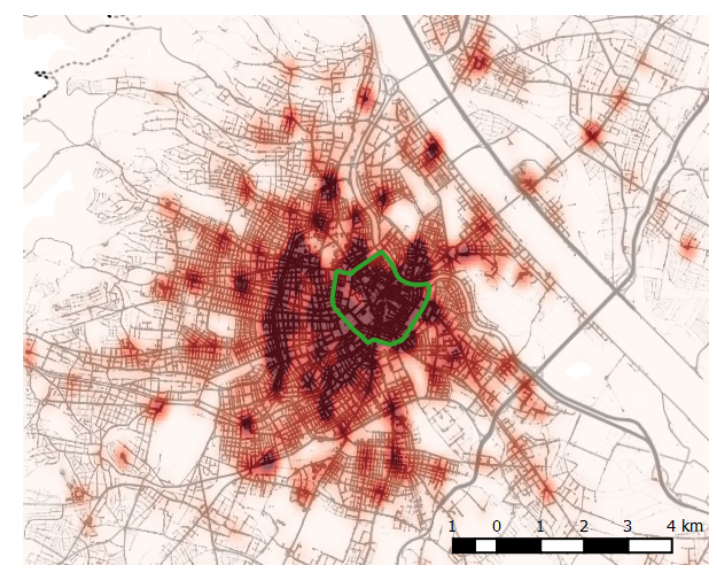

Figure 2: Start and end locations of trips in Vienna, Austria, as density map, where dark red indicates a high value. The green bold line encloses the city center.

chosen by the customer and therefore the frequency of start/end locations should not be affected. But if customer trips to some destinations are resigned by the driver due to an insufficient driving range, less BEVs and therefore less charging demand can be expected at those destinations. However, we expect the driver to always head for a CS instead of accepting only trips to reachable destinations. In a recent study the behaviour of $600 \mathrm{BEV}$ taxis was investigated for Shenzen, China (Tian et al., 2014), where the authors concluded that the time and distance a taxi is occupied, is comparable between BEV and ICEV. But they also argue that a CS is under-utilized if it is 'far away' from other CSs. Whether a CS is isolated can be identified from the maps with isochrones (Fig. 6).

The investigation of a large fleet of taxi vehicles reveals areas with an increased number of start and end locations of trips. This can be seen in Fig. 2 where a density map of start and end locations of all trips from year 2014 is shown. All data is smoothed with a quadratic (bi-weighted) kernel and a bandwidth of $500 \mathrm{~m}$. A non-uniform distribution of locations is clearly visible.

Most customer trips start and/or end close to the city center of Vienna (indicated by bold green line). With increasing distance from the city center, a decreasing number of trip locations is visible. One reason for this is that the inner region of Vienna is more densely populated compared to the other districts. Moreover, in the city center and all neighboured districts parking space is regulated but only in some outer districts. Therefore, people tend to not use their own car to avoid parking fees. Finally, the region around city center offers above-average possibilities for free-time and touristic activities and are attracting more and also farther living people. In Fig. 2 we can also observe some "hotspots" farther away from the city center. These are mostly community facilities like hospitals or railway stations.

In areas with a larger amount of start and end locations of trips, the probability is higher that a taxi BEV starts or ends a trip. Since BEVs will more frequently arise in these regions, also the charging demand will be increased in those areas. Consequently, we choose the frequency of start and end locations as indicator for charging demands. 


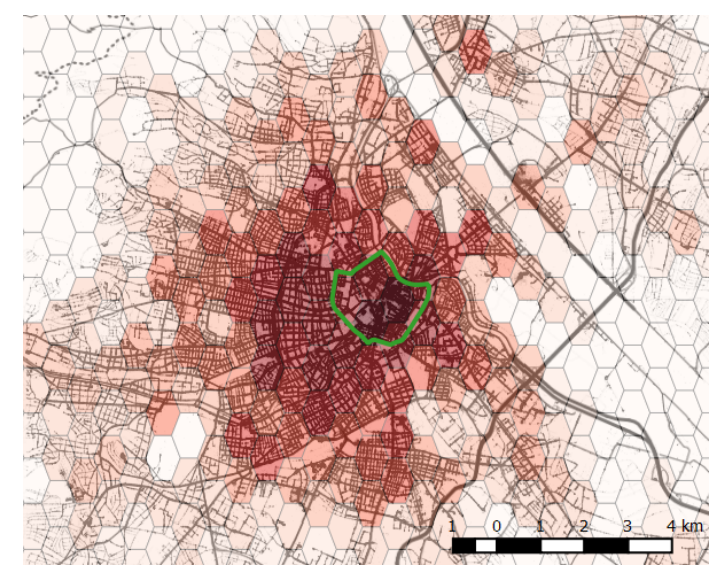

Figure 3: Hexagon grid with a colouring corresponding to the trip location counts inside the cell. Dark red indicates a high value.

\subsection{Aggregation of charging demand}

It is not an objective to find exact locations for placing CSs. Instead, the outcome should be regions, within which a placement is reasonable. Therefore, the study area is subdivided into uniform cells, where each cell may contain a CS. The spatially distributed charging demand is aggregated, meaning that start and end locations of taxi trips within each cell were summed up. In case a cell contains a CS, it covers the charging demand of the corresponding cell, independent where exactly the CS is placed. This is only possible if travel times and distances within a cell are low. Therefore, the diameter of a cell is chosen to be one kilometre, which results in a maximum travel time of 4 minutes, given an average European urban travel speed of $15 \mathrm{~km} / \mathrm{h}$ (Jensen et al., 2010). Also, in a large cell the chance is higher to find a suitable location for a CS. Therefore, a diameter of one kilometre is considered to be a good trade-off between keeping the chance of finding a suitable location high and travel times low. A CS in a cell not only covers the demand of the same cell but also with a certain weight the demand of its neighbours. Therefore, the shape of a cell was chosen to be hexagonal because in contrast to triangles or squares the distances to all neighboured hexagons are equal. Additionally, by using hexagons instead of circles a complete tessellation of the investigated region is possible.

\subsection{Selection of optimal regions}

To optimally select regions for placing CSs based on the aggregated demands we formally define the following optimization problem based on the maximal covering location problem (Church and ReVelle, 1974): The total region is partitioned into a set of hexagons $H$. Each hexagon is assigned a value $c_{i}, i \in H$, counting the taxi trips starting or ending within this hexagon. Furthermore, we denote the hexagons which have a direct connection to a hexagon $i \in H$ as set of neighbours $N_{i} \subseteq H \backslash\{i\}$. A hexagon $i$ is covered with weight $w_{0} \in[0,1]$ if $i$ itself is selected for placing a CS or with weight $w_{1} \in[0,1]$ if a neighbour in $N_{i}$ contains a CS. The subset $\bar{H} \subseteq H$ denotes the set of hexagons which already contain a CS. The number of new CS to be built is limited by $R$ and is defined by the planner and investor. The decision for a specific value for $R$ is based on 
budget constraints and requirements of the drivers. In Section 4.2 we discuss the impact of different values for $R$ on the coverage of charging demand and on the accessibility of CS.

The aim is to maximize the sum of covered trip counts, whereas (due to the weighting) a region can only be covered at most $M>0$ times. Although the model can deal with arbitrary positive values of $M$, we set $M=1$ since higher values would lead to a higher density of CSs near regions with large $c$-values.

To solve the problem at hand we use the methodology of mixed-integer linear programming (MILP) (cf. Nemhauser and Wolsey, 1988) because of the following reasons: (i) It easily allows variations in the coverage constraints of the basic maximal covering location problem, (ii) we can solve the resulting model with an off-the-shelf MILP solver, and (iii) the consideration of existing facilities can be managed by simply fixing variables in the model.

We use continuous variables $x_{i} \geq 0, \forall i \in H$, to represent the number of times hexagon $i$ is covered in the solution. Binary variables $y_{i} \in\{0,1\}, \forall i \in H$, decide if a recharging station is located in region $i$ or not. The resulting MILP model can be written as:

$$
\begin{array}{rlrl}
\max \sum_{i \in H} c_{i} x_{i} & & \\
\text { subject to } \sum_{i \in H \backslash \bar{H}} y_{i} & \leq R & \\
y_{i} & =1 & & \\
x_{i} & \leq w_{0} y_{i}+\sum_{j \in N_{i}} w_{1} y_{j} & & \forall i \in \bar{H} \\
0 \leq x_{i} & \leq M & & \forall i \in H \\
y_{i} & \in\{0,1\} & & \forall i \in H \\
& & \forall i \in H
\end{array}
$$

Objective function (1) maximizes the sum of covered taxi trip counts. Constraints (2) limit the number of new CSs to be built by $R$, and equations (3) fix all existing stations to 1 . Cover constraints (4) ensure that a region $i$ is only covered (with according weights) if at least $i$ or one of its neighbours is selected for placing a CS. Constraints (5) and (6) define the feasibility ranges of the used variables.

Since the model has a quite simple structure and the instances at hand are rather small, we solve it exactly by using the MILP solver IBM ILOG CPLEX 12.6.1. The model for the Vienna city region with about 1500 hexagons, i.e., $|H| \approx 1500$, can be solved within one second. We vary $R$ in the range from 5 to $30 \mathrm{CS}$, fix weight $w_{0}=1$ and choose $w_{1}$ from $\{0.5,1\}$.

\subsection{Selection of detailed locations}

The final steps to select appropriate positions for charging points within the selected regions are based on stakeholder decisions regarding the local planning and installation of charging infrastructure. Main considerations are based on:

- local prospects and possibilities

- economics and partnerships

- consumer acceptance and appearance 
- expandability

The hexagons selected by the optimization algorithm represent a basic concept in an appropriate grid size for the stakeholder consultations. To decide on local prospects and possibilities experts may analyse possible real estates, the actual electric power grid and expandability as well as the transport connection. From the economic point of view the partnerships for installation and operation of charging infrastructure are of crucial importance. To ensure consumer acceptance, taxi companies and drivers take part in the stakeholder process as well as the city of Vienna supporting design, appearance, and marketing.

A CS placed at a specified location covers the charging demand of all vehicles in the vicinity (defined by parameter $w_{0}$ and $w_{1}$ ). Charging demand is based on the frequency of start/end locations and therefore an increased taxi density is expected for these regions. In order to avoid a competition among drivers for the same charger, the number of charging points is continuously increased, where the decision for an expansion is based on the occupancy of the CS. Since in a first phase only 40 taxi BEVs are purchased, only little competition among drivers is expected in the beginning.

\section{Results}

In this section we present the optimization results for a CS infrastructure for taxi BEVs in Vienna, Austria. In a preliminary analysis we show that BEVs are qualified as taxi cabs and argue for fast charging stations as most suitable for a taxi service. Charging demand is estimated on the basis of real taxi data of the year 2014 and contains approximately 6.3 million trips. Based on this charging demand we identify areas for placing the CSs. Further, we compare different scenarios with a varying number of CSs and investigate the influence on road network coverage.

\subsection{Preliminary analysis}

The currently higher acquisition costs of BEVs compared to internal combustion engine vehicles (ICEVs) are compensated by lower operational energy costs. Moreover, the drivetrain of a BEV contains less rotating and moving parts (e.g., no clutch or injection, only single gear transmission), which results in lower expenses for maintenance. According to Kampker et al. (2013) the total cost of ownership (TCO) of a Smart Fortwo ICEV exceeds the TCO of a comparable BEV after approximately eight years, assuming an annual mileage of $10,000 \mathrm{~km}$. This assessment depends on the development of energy costs but it can be assumed that for the next decades the price of fossil energy will significantly exceed the price of electric energy. In the study by Kampker et al. (2013) the annual mileage has been assumed quite small while for a typical taxi vehicle in Vienna, Austria, an annual mileage of 40,000 km and more is usual. Therefore, the point of break even of a taxi BEV compared to an ICEV can be expected within two years or less.

Although driving patterns of taxis are advantageous for introducing BEVs, there are some peculiarities which have to be considered. Many taxi vehicles are operated $24 / 7$, meaning that several drivers share one vehicle over different shifts. Since nearly no time is left between two shifts, there is not sufficient time left for level I (slow) charging, which is the most common charger used for private 
Table 1: Charging station terminal types

\begin{tabular}{|c|c|c|c|}
\hline Level & Type & Power $[\mathrm{kW}]$ & Duration $[\mathrm{h}]$ \\
\hline \hline I & Slow charging & $1.92(\mathrm{AC})$ & $>10$ \\
\hline II & Standard charging & $2.5-19.2(\mathrm{AC})$ & $6-8$ \\
\hline III & Fast charging & $<240(\mathrm{DC})$ & 0.5 \\
\hline
\end{tabular}

purposes. In Table 1 different CS terminal types are listed, including estimated charging times for a battery size of $24 \mathrm{kWh}$. As shown, charging durations for level I and II chargers are several hours. Using level I or II chargers would either imply downtimes for several hours or only a relatively small fraction of the battery capacity can be charged during intermediate waiting times. Long downtimes are only possible for vehicles not used 24/7 and drivers who are willing to quit work after the battery is empty. Incomplete charging however would force the driver to search for a CS several times a day as well as to plug and unplug each time. Due to average waiting times of approximately 20 minutes (compare Fig. 4c) charging at level II has to be initiated approximately 18 times to drive the distance of a fully charged battery. We also could resign from using level III CSs, therefore reduce installation costs and purchase a replacement vehicle (BEV or ICEV) instead. Then, the BEV is charged while the other one is in operation. In this scenario the problem is, that taxi enterprises are small scale enterprises and therefore a replacement vehicle and additional fixed expenses (insurance, maintenance, etc.) are often not affordable. Also limited parking space and the logistic effort to switch between vehicles if battery is empty (or fully charged) are downsides for this concept. In contrast to that, level III CSs will be installed (and already are installed) by an electric energy provider, who is able to bear the investment costs. CSs are financed by a small extra charge when using them, where the payback period is projected to 15 years.

In this study we consider a Nissan NV200 electric as taxi. This BEV is already used in other cities as a taxi and has according to the New European Driving Cycle (Barlow et al., 2009) tests an average range of $170 \mathrm{~km}$. Its 24 $\mathrm{kWh}$ Li-Ion battery can be charged to 80 percent in 30 minutes or less with a level III fast charger, i.e., 30 minutes fast charging results in a approximate driving range of $136 \mathrm{~km}$.

In Fig. 4 the result of the evaluation of approximately 720,000 taxi trips from year 2011 is visualized. This data is enriched with shift durations and waiting times at taxi stands, an information solely available for the data set from 2011. A trip is always starting and ending at a taxi stand. This means, in addition to the distance travelled with a customer on board, also the journey to the pickup point and back to the taxis stand are included in the trip. Fig. 4 can be interpreted as follows:

- Fig. 4 a shows that the distance of taxi trips is almost always below 136 $\mathrm{km}$. The evaluation showed that from all trips $(720,000)$ only 462 trips were above $136 \mathrm{~km}$. For the considered BEVs this is the driving range with an SOC of about $80 \%$. 


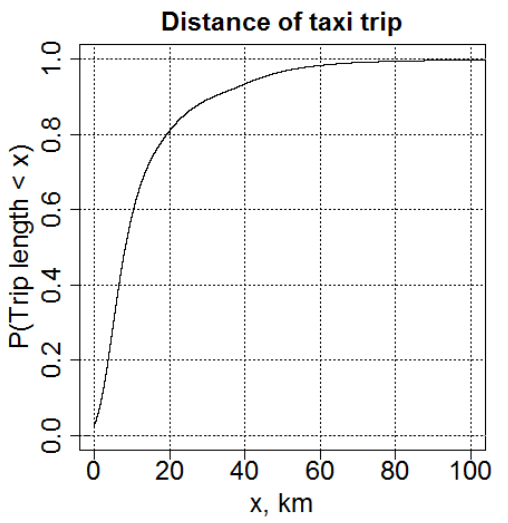

(a)

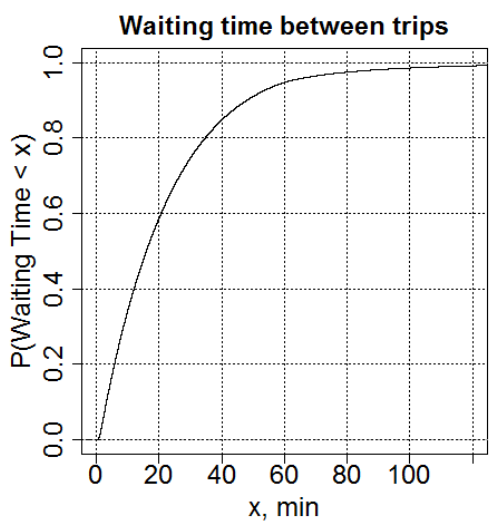

(c)

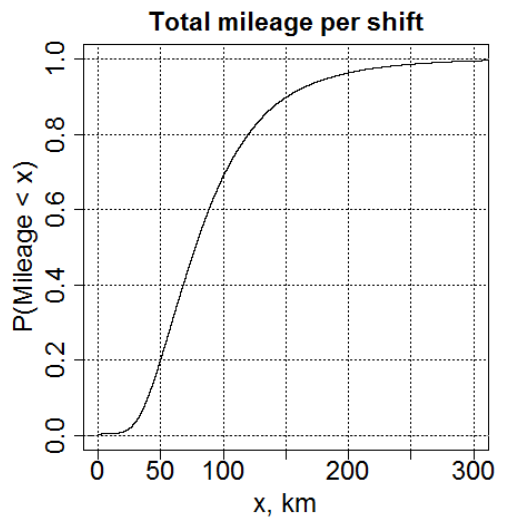

(b)

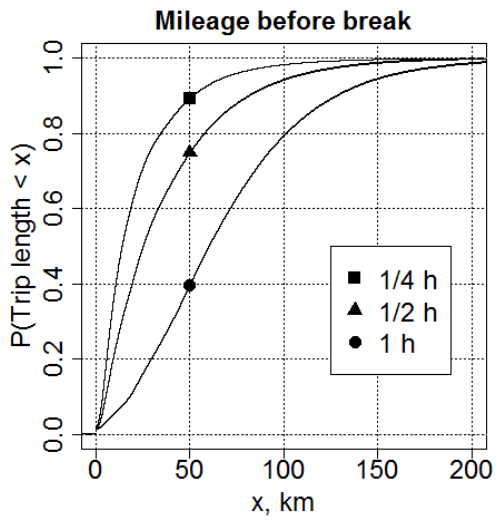

(d)

Figure 4: Probability distribution of trip length (a), total mileage per shift (b), waiting times (c), and mileage before break (d). Evaluation is based on operational data from 2011.

- The total mileage during a shift is visualized in Fig. 4b. In Section 1 we mention that the point of break even for BEVs compared to ICEVs is achieved after 2 years, based on an annual mileage of 40,000 km. If we assume the taxi BEV to be in operation for 350 days per year (considering downtimes for maintenance) and it is used for two shifts a day, then during one shift a driver has to complete $57 \mathrm{~km}$ to achieve an annual mileage of $40,000 \mathrm{~km}$. From Fig. 4b we see that this is the case for more than $75 \%$ of all shifts. Moreover, a large amount of shifts has a significant higher mileage resulting in reaching earlier the point of break even. A taxi vehicle is in operation for approximately 5 years. Therefore the annual mileage of a BEVs has to be at least $16,000 \mathrm{~km}$ to be more cost-efficient than an ICEV within the lifetime of a taxi vehicle.

- Waiting times between consecutive customer trips can be used for charging the BEV. Since approximately $95 \%$ of all waiting times are below one hour, cf. Fig. 4c, only stations for fast charging level III, cf. Table 1, make sense. 
- In Fig. 4d the total mileage of consecutive trips before a longer time break is visualized. The analysis was performed for different break durations (15, 30, and 60 minutes). If 30 minutes are considered for charging the vehicle (resulting in a SOC of $80 \%$ ), approximately $98 \%$ of consecutive trips may be accomplished if the driving range is $136 \mathrm{~km}$. Thus, in most cases normal waiting times can be used for charging, without any additional dwell times.

From Fig. 4c we see also that waiting times are not sufficient for charging in many cases. Either the driver interrupts charging or he/she forgoes a customer trip. Drivers who interrupt charging and leave the CS with a partially charged battery, will have to charge earlier the next time. For the same mileage they have to charge more often and therefore the reduction of distances to the nearest CSs becomes more relevant.

If otherwise the driver decides to proceed charging, a customer trip has to be resigned. Insufficient charging time is expected more probably for locations with a high customer frequency. But at these locations a new customer can be found shortly after charging has been finished. The maximum number of 'lost' customer trips depends on the ratio between charging time and average customer trip duration. From our data we obtain an average customer trip duration of 24 minutes, including the travel time to the customer and back to a taxi stand. This means during 30 minutes of charging, only one customer trip cannot be accomplished. The number of resigned customer trips at a CS could be higher (or lower) depending on the customer frequency.

On the basis of this evaluation we argue that BEVs are appropriate for replacing ICEV taxis, assuming similar specifications as the considered vehicles (e.g., Nissan NV200 electric). High mileages lead to an early point of break even compared to ICEVs and driving behaviour is proper for using intermediate charging, but only if an infrastructure for fast charging is available. Therefore, it can be expected that the influence of charging on taxi operation is small and financial losses due to missed passenger trips are negligible.

Alternatively to fast CSs battery swap stations (BSSs) may be considered. With a BSS it is possible to reduce charging times to a few minutes. However, this type of charging infrastructure is generally more suitable for highways (Baster et al., 2013) and has some major drawbacks:

- The costs of a BSS are about ten times the costs of a fast CS (Baster et al., 2013). If we decide to place one BSS instead of ten fast CSs, distances and travel times to the next BSS/CS will increase which alleviates the advantage of low charging times.

- A certain amount of replacement batteries has to be stored at each BSS. This would further increase the system costs.

- A BSS demands more space compared to a fast CS because of the construction for swapping and battery storage facilities. Moreover, for the battery storage facility constructional safety rules have to be adhered in order to mitigate the risk of fire. Thus, a BSS is more likely to be placed at the periphery of Vienna and therefore would further increase trip distance and travel time to a CS. 
- For many vehicles (e.g., Nissan e-NV200) the battery cannot be simply swapped by a BSS. In general, swapping batteries requires a stringent standardization of batteries.

A business model to establish BSSs developed by Better Place failed in 2013 (Kershner, 2013) and currently it is not possible to purchase a working BSS for a service as pursued in this study. Also Tesla recently decided to resign from BSSs for their BEVs. Due to this and the above mentioned reasons we conclude that a charging infrastructure with BSSs is not feasible for the city of Vienna.

\subsection{Optimization results}

For the optimization process the quantity $R$ of CSs and the weights $w_{0}, w_{1}$ have to be fixed a priori. To demonstrate the effect of different parameter settings we perform the optimization for different values of $R$ and $w_{1}$ while fixing $w_{0}=1$. Three already existing fast CSs (level III) in Vienna are considered in the problem formulation. Fig. 5 shows the optimization results. Red triangles represent selected hexagons where the placement of a CS is recommended and black stars are hexagons with an already active CSs. Increasing weight $w_{1}$ and keeping $R$ constant causes an increased scattering of CS locations over the urban area. We do not consider $w_{1}=0$ since then only the $R$ hexagons with highest demand would be selected.

We create maps with isochrone for all solutions from Fig. 5 in order to demonstrate the accessibility of the proposed charging infrastructure. For this analysis the exact location is assumed to be in the center of the hexagon. The influence of placing the CS not in the center of a hexagon, is analysed later in this section. In the maps of Fig. 6 all road links are highlighted from which a CS can be reached within a certain time limit. Travel times used in the calculation of isochrone are based on taxi floating car data, (cf. Toplak et al., 2010), and represent average values for working days at 8 am (morning rush hour). This corresponds to a rather pessimistic assessment of accessibility. We perform the analysis for two different time limits, i.e., five and eight minutes.

In Fig. 6 the hexagon grid is also superposed. It can be seen that for a travel time up to five minutes the whole road network inside a hexagon with a CS is accessible and large fractions of neighboured cells. Within eight minutes travel time all roads in neighboured hexagons are accessible. This result supports the modelling approach to cover also the demand of neighboured cells by a CS. 


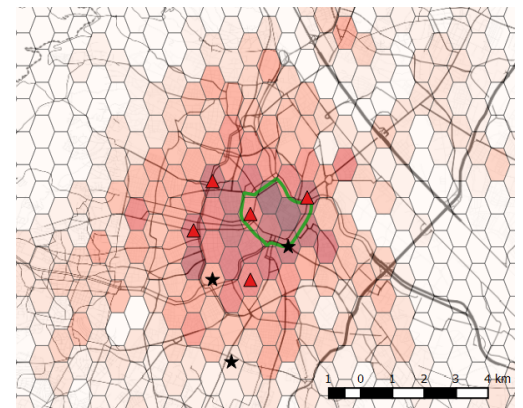

(a) $R=5, w_{1}=0.5$

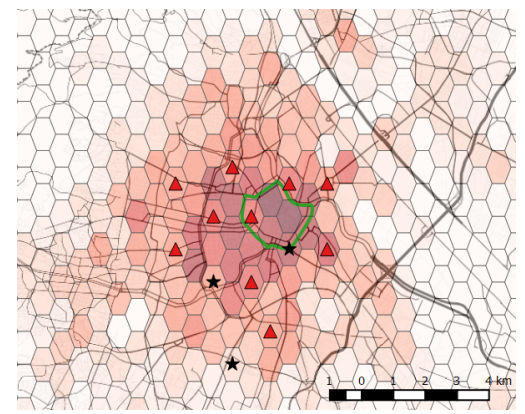

(c) $R=10, w_{1}=0.5$

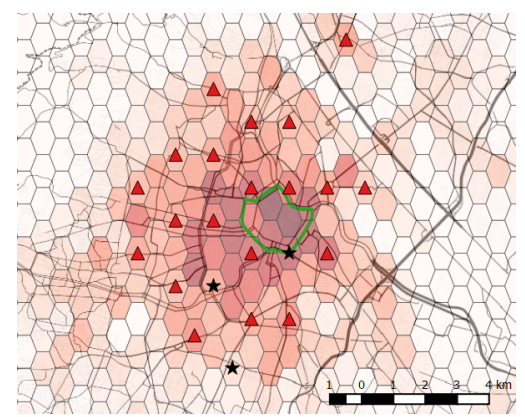

(e) $R=20, w_{1}=0.5$

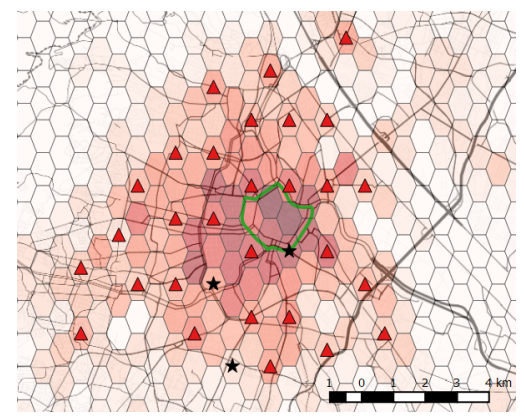

(g) $R=30, w_{1}=0.5$

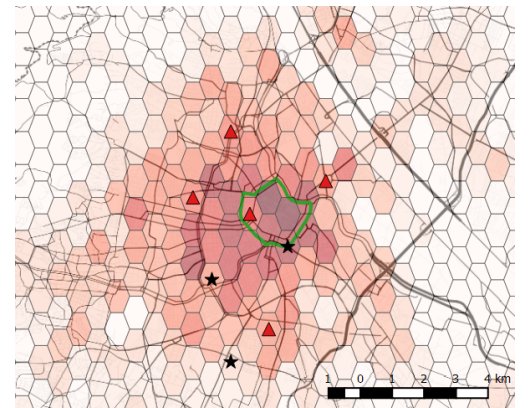

(b) $R=5, w_{1}=1$

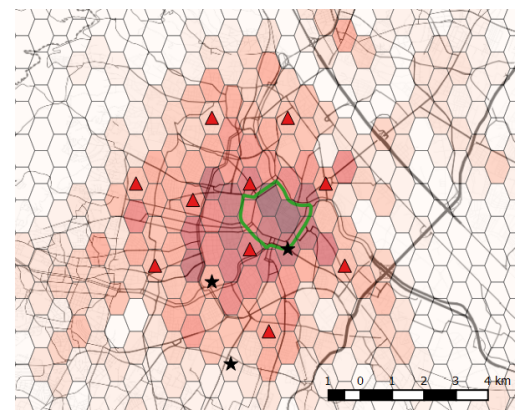

(d) $R=10, w_{1}=1$

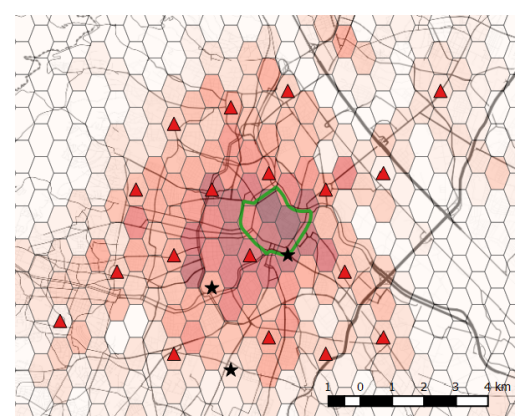

(f) $R=20, w_{1}=1$

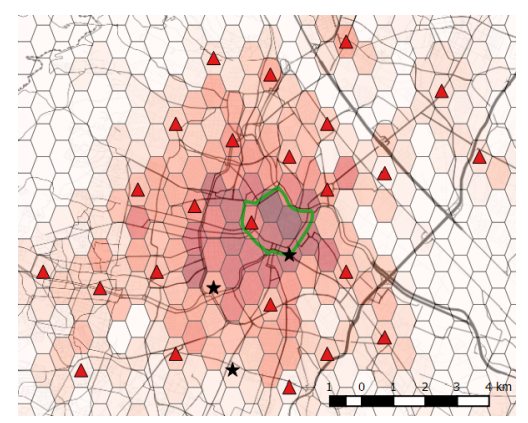

(h) $R=30, w_{1}=1$

Figure 5: Optimized locations for different $R$ (number of CS) and $w_{1}$ (weight for neighboured cell,) represented as triangles. Existing CSs are visualized by stars. For $R=20$ and $R=30$ not all locations are visible on the presented map detail. 


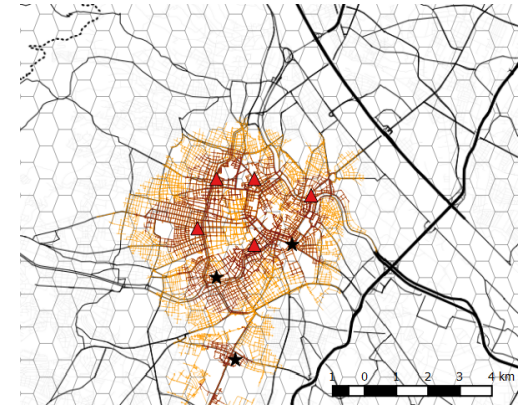

(a) $R=5, w_{1}=0.5$

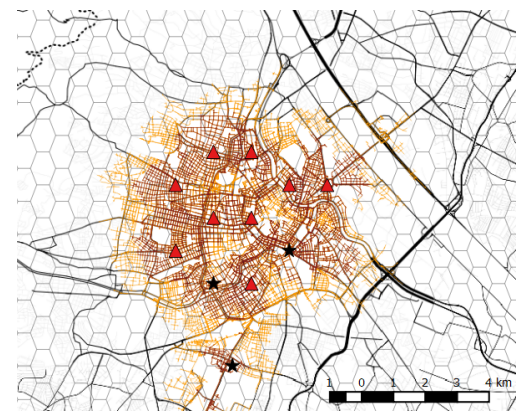

(c) $R=10, w_{1}=0.5$

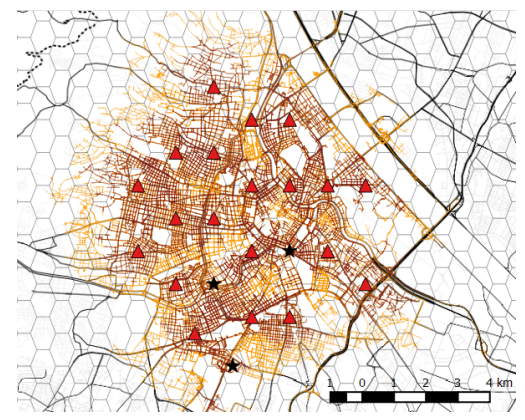

(e) $R=20, w_{1}=0.5$

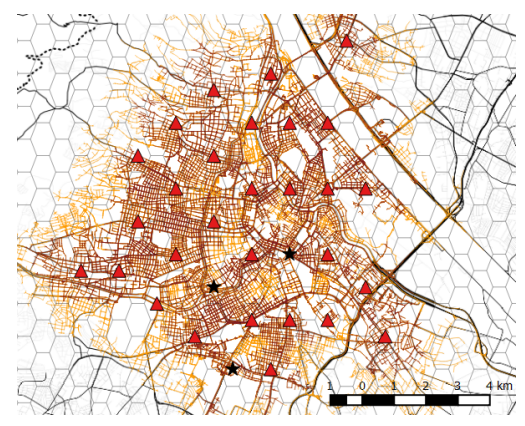

(g) $R=30, w_{1}=0.5$

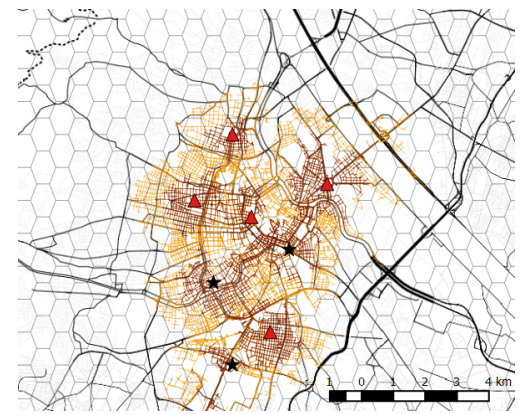

(b) $R=5, w_{1}=1$

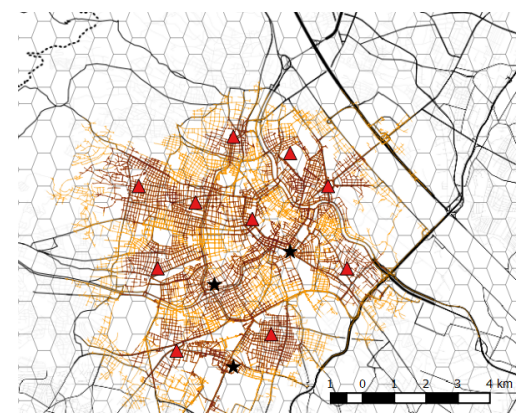

(d) $R=10, w_{1}=1$

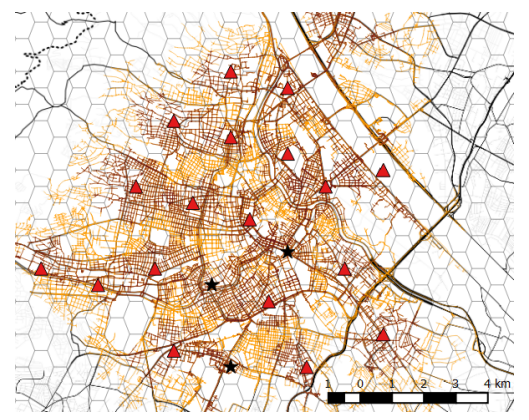

(f) $R=20, w_{1}=1$

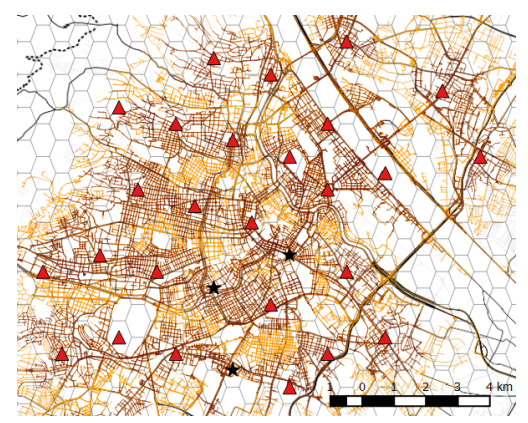

(h) $R=30, w_{1}=1$

Figure 6: Road links within five (red) and eight (yellow) minutes distance to a CS.

Results of the isochrone are further used to estimate the coverage of the 
Table 2: Minimum and maximum percentage of network length within five and eight minutes distance to a CS. The total network length is $3807 \mathrm{~km}$.

Percentage of network length (\%)

\begin{tabular}{ccrr}
\hline & & Travel time $\leq 5$ min & Travel time $\leq 8$ min \\
\hline$R$ & $w_{1}$ & [Min., Max.] & [Min., Max.] \\
\hline 5 & 0.5 & {$[9,10]$} & {$[21,24]$} \\
5 & 1 & {$[11,13]$} & {$[28,31]$} \\
10 & 0.5 & {$[16,17]$} & {$[31,35]$} \\
10 & 1 & {$[18,20]$} & {$[38,43]$} \\
20 & 0.5 & {$[28,30]$} & {$[49,53]$} \\
20 & 1 & {$[30,35]$} & {$[68,73]$} \\
30 & 0.5 & {$[40,42]$} & {$[70,75]$} \\
30 & 1 & {$[41,47]$} & {$[93,99]$}
\end{tabular}

road network (ignoring the demand) and the sensitivity of selecting the exact location. Instead of assuming the exact location of a CS in the center, this time it is chosen randomly and independently within the hexagon. This step is repeated ten times for each solution and according isochrones are calculated for each sample. I.e., for each solution obtained from the optimization, we get ten different network coverage estimates. Table 2 lists the fraction of the entire road network with a travel time up to five and eight minutes to a CS for different parameter settings. For each set of parameters the minimum and maximum coverage as obtained from our samples is listed. Since differences between minimum and maximum are small (up to six percentage points), the sensitivity of selecting the exact location on network coverage is small as well.

For a fixed number of CSs and five minutes travel time, parameter $w_{1}$ has only little influence on coverage. This is not the case for eight minutes travel time, where e.g., coverage increases by approximately 20 percentage points for 30 CSs when increasing $w_{1}$. Moreover, an almost complete coverage $(>93 \%)$ for a travel time up to eight minutes is possible with $30 \mathrm{CS}$.

Clearly, as the number of CS increases a larger area is covered and from more road links a CS is reachable within a certain travel time. The demand coverage is depicted in Fig. 7 for different numbers of CSs and values for $w_{1}$. Coverage is defined as the relative total count (start and end locations) inside or directly next to a hexagon containing a CS. This definition is supported by the maps with isochrone in Fig. 6 where all road links of neighboured hexagons are reachable within five to eight minutes travel time.

For higher numbers of CSs a non-linear coverage increase can be observed. Note that a saturation of the charging demand can be seen in Fig. 7, beginning from $20 \mathrm{CSs}$. When decreasing $w_{1}$ coverage decreases since CS locations are closer together. Although the final decision of the number of CS to be installed depends on budget constraints, our evaluation may support this decision.

\section{Conclusions}

In this study we presented a method for finding optimal regions for placing a predefined number of CSs. The objective was to maximally satisfy the charging demand of taxi BEVs. Start and end positions of customer trips of a large 


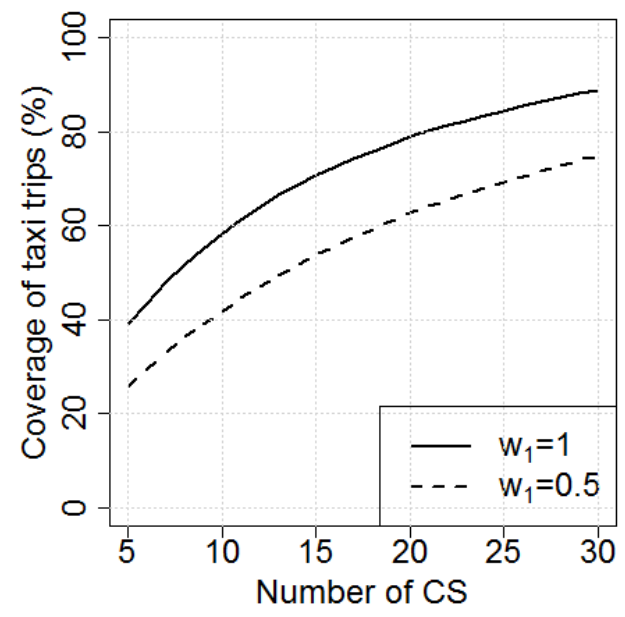

Figure 7: Percentage of covered charging demand subject to number $R$ of CSs and weight $w_{1}$ $\left(w_{0}=1\right)$.

fleet of conventional taxis were aggregated to areas (represented as hexagons). The amount of positions inside a cell was considered as charging demand. This estimation of charging demands was chosen to minimize driving distances from a CS to the pickup point of a customer and vice versa. The demand inside a hexagon may not only be covered by a CS in the same cell but also partially by a CS in a neighboured cell. Moreover, the optimization problem allows the integration of already existing CSs, enabling a iterative development of the charging infrastructure. Finally, the simple structure of the optimization problem allows an exact solution. The result is a set of areas instead of specific road links or intersections, in which the exact locations for CSs have to be defined by human inspectors on the basis of environmental constraints. In this sense the method is a decision support system which has been applied during a research project with the aim of introducing taxi BEVs in Vienna, Austria.

For Vienna no experience regarding the interdependency between charging infrastructure and driving behaviour is available. This will be investigated further as soon as CSs are implemented and BEVs are in operation. In a first phase $40 \mathrm{BEVs}$ will be purchased and therefore only little competition for the same CS among drivers is expected. However it is a topic for future research activities to optimize the expansion of different CSs by additional charging points, to allow more than one BEV to be charged.

\section{Acknowledgements}

We gratefully thank Taxi 31300 (Taxi 31300 Vermittlungs-GmbH) for providing the taxi data used in this study. The work was done within the Austrian research project W-ETaxi, co-funded by the Austrian Climate and Energy Fund (KLiEn) under the first phase of the programme: "E-Mobility for all: Urban E-Mobility" (Project Nr. 845081). Further this this work was partially funded by the Austrian Federal Ministry for Transport, Innovation and Technology 
(BMVIT) within the strategic program ERANET Electromobility+ under the grant 832423 (SELECT - Suitable Electromobility for Commercial Transport) and JPI Urban Europe under the grant $847350\left(e^{4}\right.$-share - Models for Ecological, Economical, Efficient, Electric Car Sharing).

\section{References}

Andrews, M., Dogru, M., Hobby, J., Jin, Y., Tucci, G., 2013. Modeling and optimization for electric vehicle charging infrastructure, in: Proceedings of Production and Operations Management Society (POMS).

Barlow, T.J., Latham, S., McCrae, I.S., Boulter, P.G., 2009. A Reference Book of Driving Cycles for Use in the Measurement of Road Vehicle Emissions. Technical Report. TRL - Transport Research Laboratory, Wokingham, Berks, UK.

Baster, H., Bentzen, K., Laugesen, M.S., Kapitanovic, E., Moller, M., Gvozdareva, I., 2013. Danish Experiences in Setting up Charging Infrastructure for Electric Vehicles with a Special Focus on Battery Swap Stations. Technical Report. FDT - Danish Association of Transport and Logistic Centres.

Cai, H., Jia, X., Chiu, A.S., Hu, X., Xu, M., 2014. Siting public electric vehicle charging stations in beijing using big-data informed travel patterns of the taxi fleet. Transportation Research Part D: Transport and Environment 33, $39-46$.

Chen, T.D., Khan, M., Kockelman, K., 2013. Electric vehicle charging station location problem: A parking-based assignment method for seattle, washington, in: Proceedings of the $92^{\text {nd }}$ Annual Meeting of the Transportation Research Board.

Church, R., ReVelle, C., 1974. The maximal covering location problem. Papers in regional science $32,101-118$.

Daskin, M.S., 2011. Network and discrete location: models, algorithms, and applications. John Wiley \& Sons.

Egbue, O., Long, S., 2012. Barriers to widespread adoption of electric vehicles: An analysis of consumer attitudes and perceptions. Energy Policy 48, 717729 .

ElBanhawy, E.Y., Dalton, R., Shankar, V.N., Warith, K.A., 2014. Hybrid-od matrix based simulation approach to identify e-charging hotspots in transport network, in: IEEE Transportation Electrification Conference and Expo (ITEC), pp. 1-6.

Gao, Y., Guo, Y., 2013. Optimal planning of charging station for phased electric vehicle. Energy and Power Engineering 5, 1393-1397.

Ge, S., Feng, L., Liu, H., 2011. The planning of electric vehicle charging station based on grid partition method, in: International Conference on Electrical and Control Engineering (ICECE), pp. 2726-2730. 
He, F., Wu, D., Yin, Y., Guan, Y., 2013. Optimal deployment of public charging stations for plug-in hybrid electric vehicles. Transportation Research Part B: Methodological 47, 87-101.

Hess, A., Malandrino, F., Reinhardt, M.B., Casetti, C., Hummel, K.A., Barceló-Ordinas, J.M., 2012. Optimal deployment of charging stations for electric vehicular networks, in: Proceedings of the First Workshop on Urban Networking, ACM, New York, NY, USA. pp. 1-6.

Jensen, P., Rouquier, J.B., Ovtracht, N., Robardet, C., 2010. Characterizing the speed and paths of shared bicycle use in lyon. Transportation Research Part D: Transport and Environment 15, 522-524.

Jung, J., Jayakrishnan, R., Choi, K., 2012. Shared Taxi Operations with Electric Vehicles. Technical Report. Institute of Transportation Studies, University of California, Irvine, CA.

Kampker, A., Schnettler, A., Vallèe, D., 2013. Elektromobilität (Electromobility). Springer.

Kershner, I., 2013. Israeli venture meant to serve electric cars is ending its run. The New York Times. http://www.nytimes.com/2013/05/27/business/global/israeli-electric-carcompany-files-for-liquidation.html?_r=2, Accessed: 2015-11-06.

Lingfeng, K., Zifa, L., Huan, Z., 2010. Modeling algorithm of charging station planning for regional electric vehicle. Modern Electric Power 27, 44-48.

Nemhauser, G.L., Wolsey, L.A., 1988. Integer and combinatorial optimization. Wiley-Interscience.

Nie, Y.M., Ghamami, M., 2013. A corridor-centric approach to planning electric vehicle charging infrastructure. Transportation Research Part B: Methodological 57, 172-190.

Owen, S.H., Daskin, M.S., 1998. Strategic facility location: A review. European Journal of Operational Research 111, 423-447.

Reinthaler, M., Asamer, J., Koller, H., Litzlbauer, M., 2014. Utilizing mobility data to facilitate the introduction of e-taxis in vienna, in: Proceedings of the International Conference on Connected Vehicles and Expo (ICCVE).

Schuster, M., Litzlbauer, M., Reinthaler, M., 2012. Zukünftige Energienetze mit Elektromobilität - Überblick der Projektziele (future energy grids with electromobility), in: Alternativen für die Energiezukunft Europas - 12. Symposium Energieinnovation, Graz.

Sellmair, R., Hamacher, T., 2014. Method of optimization for the infrastructure of charging station for electric taxis, in: Proceedings of the $93^{\text {rd }}$ Annual Meeting of the Transportation Research Board.

Tian, Z., Wang, Y., Tian, C., Zhang, F., Tu, L., Xu, C., 2014. Understanding operational and charging patterns of electric vehicle taxis using gps records, in: Intelligent Transportation Systems (ITSC), 2014 IEEE 17th International Conference on, pp. 2472-2479. 
Toplak, W., Koller, H., Dragaschnig, M., Bauer, D., Asamer, J., 2010. Novel road classifications for large scale traffic networks, in: $13^{\text {th }}$ International IEEE Conference on Intelligent Transportation Systems (ITSC), pp. 1264-1270.

Wang, G., Xu, Z., Wen, F., Wong, K.P., 2013. Traffic-constrained multiobjective planning of electric-vehicle charging stations. IEEE Transactions on Power Delivery 28, 2363-2372.

Wang, H., Cheu, R.L., 2013. Operations of a taxi fleet for advance reservations using electric vehicles and charging stations, in: Proceedings of the $92^{\text {rd }}$ Annual Meeting of the Transportation Research Board. 